\title{
MedienPädagogik
}

Zeitschrift für Theorie und Praxis der Medienbildung

\section{Lehren und Lernen mit und über Medien in Kooperation von Schule, Hochschule und Museen}

\section{Am Beispiel des Projekts (Reuchlin digital)}

Daniel Autenrieth, Claudia Baumbusch und Anja Marquardt

\section{Zusammenfassung}

Der Beitrag stellt das Entwicklungsprojekt 〈Reuchlin digital〉 (RD) am Museum Johannes Reuchlin (MJR) in Pforzheim vor, welches im Schnittfeld von kultureller Bildung (in schulischen und ausserschulischen Kontexten), Medienpädagogik und der Lehramtsausbildung verortet ist. Johannes Reuchlin (Humanist und Zeitgenosse der Medienrevolution des Buchdrucks) setzte sich für Toleranz und die Erkundung alles Fremden ein. Seine Botschaften stehen leitmotivisch über dem Projekt. RD ist als praxisbezogenes Entwicklungs- und Modellprojekt mit evaluativen Elementen angelegt. Der Schwerpunkt des Beitrags soll auf der Darstellung theoretisch-konzeptioneller Überlegungen sowie wichtiger Zwischenergebnisse liegen. $R D$ wurde vom Kulturamt Pforzheim initiiert (Förderpartner: Innovationsfonds BW, Arbeitsstelle literarische Museen BW) mit Kooperationspartnern verschiedener Institutionen ( $\mathrm{PH}$ Ludwigsburg, Medien- und Filmgesellschaft BW, Schulen verschiedener Schularten). Ein zentrales Anliegen von RD ist es, durch die aktiv-produktive, kreative und kritisch-reflexive Nutzung digitaler Medien und insbesondere durch die Vermittlung visueller, auditiver und audiovisueller Ausdrucksformen neue Lernwege zu eröffnen und einen ausserschulischen Lernort unter Einbezug kultureller, ästhetischer und medialer Aspekte handlungsorientiert zu erschliessen. 


\title{
Teaching and Learning with and About Media in Cooperation with Schools, University and Museum
}

\begin{abstract}
This article introduces the development project 'Reuchlin digital' (RD). The project aims for the further development of 'Museum Johannes Reuchlin' (MJR) in the southern German town of Pforzheim. MJR is a museum dedicated to the humanist Johannes Reuchlin who lived during the time of the invention of printing. Johannes Reuchlin advocated tolerance and stood for the exploration of the unknown. These principles are guiding project development. $R D$ works at the intersection of cultural education (in both school and extracurricular activities), media education and teacher training. The project is related to practice but includes evaluative elements leveraging both qualitative and quantitative methods. The article aims to outline theoretical and conceptual aspects of $R D$ and conveys important preliminary results. $R D$ resides at the Pforzheim cultural office and is funded by the city itself, the Baden-Wuerttemberg innovation fund, the 'Arbeitsstelle literatische Museen BW' and cooperates with different institutions (University of Education Ludwigsburg, Medien- und Filmgesellschaft BW and a number of schools). One of the main project goals is the exploration of new and action-oriented learning paths guided by creative, critical and reflexive methods of media production for the museum as an extracurricular learning environment. Visual, auditory and audio-visual expressions are playing a key role in this cultural, aesthetic and media related appropriation of the museal space.
\end{abstract}

\section{Das Museum Johannes Reuchlin und die Stadt Pforzheim}

Ausgangspunkt für das Projekt 〈Reuchlin digital〉 (RD) ist das seit elf Jahren bestehende Museum Johannes Reuchlin (MJR) an der Schlosskirche in Pforzheim und dessen Vermittlungsarbeit für Schulklassen im Rahmen der Kulturellen Bildung des Kulturamts.

Das MJR ist dem 〈grossen Sohn Pforzheims〉, dem Humanisten Johannes Reuchlin (1455-1522) als 〈grossem Sohn Pforzheims〉 gewidmet. Als Anbau an die Schlosskirche greift das Museum im Aussenbau die 
Formsprache der einstigen Sakristei auf, die bis zur Zerstörung des Gotteshauses im Zweiten Weltkrieg bestand. In dieser Sakristei wurde nach Reuchlins Tod, gemäss seinem letzten Willen, seine kostbare Bibliothek für einige Jahrzehnte aufbewahrt, danach weit verstreut. Etliche Bände befinden sich heute in der badischen Landesbibliothek. Im modernen Inneren des Museums wird auf vier Ebenen Leben, Werk und Wirken Reuchlins dokumentiert.

\subsection{Johannes Reuchlin und seine Botschaften als Leitmotiv für das Projekt}

«Johannes Reuchlin ist unzweifelhaft der berühmteste Sohn der Stadt Pforzheim.» (Sturm 2018, 6) Reuchlin lebte an einer Zeitenwende: vom Mittelalter zur Neuzeit und Renaissance. Als promovierter Jurist zählte der dank seiner Sprachbegabung häufig in diplomatischen Diensten durch Europa reisende Humanist zu den ersten, welche die Segnungen des Buchdrucks als Folge einer umwälzenden Medienrevolution erlebten und betätigte sich gelegentlich selbst als Verleger. Mit seinen sprachwissenschaftlichen Studien zur lateinischen und v.a. griechischen Sprache beförderte Reuchlin die Reformation Luthers, auch wenn er selbst zeitlebens Gegner des Protestantismus und überzeugter Katholik geblieben ist. Gleichzeitig nahm er eine durchaus kritische Haltung, etwa in Ablass-Angelegenheiten, gegenüber Papsttum und der Kirche ein. In späteren Jahren widmete er sich intensiv dem Hebräisch-Studium (vgl. Schwab 1998).

Als Kaiser Maximilian 1511 eine Studie in Auftrag gab, ob «der Juden Bücher verbrannt werden sollten» (Timm 2018, 24). äusserte sich Reuchlin als einziger einer Reihe von Gutachtern mit der Maxime «Verbrennt nicht, was Ihr nicht kennt.» (ebd.) In seinem Rechtsgutachten, dem er den Titel «Augenspiegel» gab, plädierte er als Jurist und Humanist. «Wenn die Juden Frieden halten, dann soll man sie auch in Frieden lassen.» (ebd.) Denn aus Intoleranz und Bücherverbrennung könne «viel Schlimmeres entstehen» (ebd.). Seinen Mitchristen hielt er den Spiegel ihrer Vorurteile und ihres Nicht-Wissen-Wollens vor. Reuchlins Fazit: Keine Gewalt! Im Interesse des friedlichen Zusammenlebens, so Reuchlin, sei es geboten, religiöse und kulturelle Minderheiten in ihren Rechten zu respektieren und abweichende 
Meinungen und Glaubensinhalte mit Geduld zu ertragen («tolerare»). Keinem Herrscher stehe es zu, seine Untertanen mit Gewalt von ihrer Religion abzubringen. Die Auseinandersetzung um religiöse und kulturelle Zugehörigkeit dürfe nur mit den friedlichen Mitteln des Dialogs geführt werden. Das Gutachten Reuchlins sorgte für einen Skandal und löste unter seinen Gegnern einen regelrechten Shitstorm aus. Der zehnjährige und phasenweise lebensbedrohliche Rechtsstreit, in den Reuchlin mit dieser Haltung geriet, endete mit seiner Verurteilung zu einer hohen Geldstrafe.

«Erkundet das Fremde, zerstört es nicht:» (ebd.) Mit seiner humanistischen Grundeinstellung setzte Reuchlin die «Brille der prüfenden Vernunft auf» (ebd.). Der Titel seines Gutachtens «Augenspiegel» war programmatisch gemeint.

Hier zeigt sich die noch immer andauernde Gültigkeit der Botschaften Reuchlins. Sie haben in der jüngsten Vergangenheit an Aktualität hinzugewonnen, Stichwort: hate speech, fake news. Als Anwalt für Toleranz und Menschenrechte hat Reuchlin ethische Grundsätze skizziert, die auch heute, in Zeiten des demografischen Wandels und einer zunehmend diversen Gesellschaft, von elementarer Bedeutung sind. (vgl. Assmann 2017, 144 ff.) «Er sprach Themen an, die auch heute geläufig sind: Migration und kultureller Austausch, Fanatismus versus Dialog, das Eigene und das Fremde, die Rolle des Staates als Beschützer des Rechts.» (Timm 2018, 25) Diese Botschaften Reuchlins stehen leitmotivisch über dem Projekt.

\subsection{Reuchlins Geburtsstadt Pforzheim}

Pforzheim zählte 2019 knapp 130.000 Einwohner. In der nach schwerster Kriegszerstörung in den Nachkriegsjahrzehnten wiederaufgebauten Stadt leben Bürgerinnen und Bürger aus über 136 verschiedenen Nationen zusammen. Pforzheim hat - bezogen auf Baden-Württemberg - den höchsten - und bezogen auf die Bundesrepublik - einen sehr hohen Anteil an Bürgerinnen und Bürgern mit einer Zuwanderungsgeschichte, aktuell 56 Prozent. Bei den unter Achtzehnjährigen liegt der Anteil bei rund 75 Prozent, bei den unter Dreijährigen inzwischen rund 90 Prozent (vgl. Stadt Pforzheim 2019, 59). Die Stadtgesellschaft setzt sich aus vielen verschiedenen Kulturen zusammen, deren friedliches und tolerantes Miteinander 
kontinuierlich thematisiert und verhandelt werden muss. Denn es gilt, den Frieden der Stadtgesellschaft zu wahren, ein Auseinanderdriften der Kulturen in «closed communities» und Parallelgesellschaften zu vermeiden und Diversität, statt Differenz zu ermöglichen. Hier ist auch die Kulturelle Bildung mit ihren Grundsätzen, Methoden und mit ihrer Förderung kultureller und gesellschaftlicher Teilhabe gefragt.

\section{Ausgangsüberlegungen, Ausgangssituation, Dimensionen und Ziele des Projekts}

\subsection{Theoretisch-konzeptionelle Überlegungen}

Unterhaltung, Kreativität, Fortbewegung, sogar Partnerschaft, Gesundheit und Wohlbefinden sind nur einige der Lebensbereiche, die von digitalen Medien, Software und Daten massgeblich bestimmt werden. Das Digitale stellt damit etwas Omnipräsentes dar. Auf der Basis von Servern, Endgeräten, Leitungen und Software ist das Digitale aber weit mehr als eine Ansammlung von Nullen und Einsen. Vielmehr ergeben sich aus den Strukturen kommunikative-soziale (vgl. Jörissen 2016, 1) kreative und auch ökonomische Möglichkeitsräume, die Menschen auf unterschiedlichen Ebenen und damit auch auf einer kulturellen Ebene berühren. Der Prozess, den wir auch als Teil der Gesellschaft durchleben, kann als Metaprozess, also als Prozess von Prozessen verstanden werden, der weder räumlich noch zeitlich in seinen sozialen und kulturellen Auswirkungen begrenzt ist (vgl. Krotz 2007, 11). Im bildungspolitischen Kontext wurde diesen Veränderungen in den letzten Jahren auf unterschiedliche Weise Rechnung getragen. Mit dem Beschluss «Medienbildung in der Schule» legte die Kultusministerkonferenz ein Papier vor, welches Medienbildung ebenfalls als einen Prozess herausarbeitet, der dauerhaft, pädagogisch strukturiert und begleitend angelegt sein soll, um sich sowohl konstruktiv als auch kritisch mit der Medienwelt auseinanderzusetzen (vgl. KMK 2012, 3). Mit der Strategie zur «Bildung in der digitalen Gesellschaft» wurde im Jahr 2016 ein weiterer Beschluss vorgelegt, welcher mit Beginn des Schuljahres 2018/2019 sukzessive umgesetzt werden sollte. Die von der Kultusministerkonferenz 
formulierten Kompetenzen (Suchen, Verarbeiten und Aufbewahren; Kommunizieren und Kooperieren; Produzieren und Präsentieren; Schützen und sicher Agieren; Problemlösen und Handeln; Analysieren und Reflektieren) sollten bis zum Ende der Pflichtschulzeit von allen Schülerinnen und Schülern erworben worden sein. Diese Bemühungen in Richtung einer fächerintegrativen und fächerübergreifenden Umsetzung von Medienbildung können zunächst positiv bewertet werden. Kritisch betrachtet werden muss aber der von der Kultusministerkonferenz verwendete Medienbildungsbegriff. Dieser wird «[sowohl] im Hinblick auf den historisch gewachsenen Medienbegriff als auch bezogen auf die Ziele verkürzt [...].» (GMK 2018, 1). Zudem fehlt in der Strategie (2016) eines der noch 2012 explizit erwähnten und zentralen Handlungsfelder: die Zusammenarbeit mit ausserschulischen Kooperationspartnern. Dazu hiess es im Beschluss «Medienbildung in der Schule» von 2012:

«Die Schule sollte zur Vermittlung von Medienbildung sowohl innerschulisch als auch an außerschulischen Orten eng und verstärkt mit anderen Institutionen und Trägern zusammenarbeiten. Dazu zählen neben Institutionen aus dem Bereich des Kinder- und Jugendmedienschutzes sowie des Datenschutzes insbesondere außerschulische Bildungs- und Kultureinrichtungen [...].» (KMK 2012, 8)

Auch wenn der Impuls zur Zusammenarbeit im Projekt «Reuchlin digital» nicht von Seiten der Schule, sondern von Seiten der Kulturellen Bildung ausgeht, ist genau diese noch 2012 von der Kultusministerkonferenz geforderte Form der Kooperation eine unabdingbare Grundkonstante des Projekts und ein wesentlicher Erfolgsfaktor. Denn gerade vor dem Hintergrund und dem Anspruch, zu einer umfassenden Medienbildung beizutragen, ist die Kooperation von Schule, ausserschulischer Kultureinrichtung und auch von Hochschule von elementarer Bedeutung. Denn eine umfassende Medienbildung kann nicht allein im Umfeld der Schule erfolgen, wo allenfalls mit und über Medien gelehrt und gelernt werden kann. Medien bestimmen aber wesentlich die Strukturen von Weltsichten, sowohl auf kultureller Ebene wie auch auf individueller Ebene: 
«Orale Kulturen, Schrift- und Buchkulturen, visuelle Kulturen und digital vernetzte Kulturen bringen jeweils unterschiedliche Möglichkeiten der Artikulation (des Denkens, des Ausdrucks, der Kommunikation, der Wissenschaften, der Künste) hervor.» (Jörissen 2013)

Diese kulturellen Möglichkeiten (auch im historischen Kontext) zu entdecken und zur Entfaltung persönlicher Stärken und Interessen nutzbar zu machen, ist auch eine Aufgabe von Medienbildung. Derartige Erfahrungswelten existieren aber vor allem ausserhalb der Schule und digitale Medien erlauben es, fast jeden ausserschulischen Ort als Lernort zu erschliessen. Er wird in einen Möglichkeitsraum verwandelt, der Lernende (nicht nur Schülerinnen und Schüler) dazu befähigt, sich in der Welt zu orientieren und diese kritisch-reflexiv und kritisch-partizipativ wahrzunehmen und sich darin und dazu zu verhalten.

Um dieses Ziel zu erreichen, muss sich das Museum als ausserschulischer Bildungsort wandeln und partizipativer werden. Lernende müssen die Möglichkeit erhalten, nicht nur Betrachterinnen oder Betrachter zu sein, sondern sie müssen zu Mitgestalterinnen und Mitgestaltern werden können (vgl. Weibel 2007, 23 ff.). Peter Weibel (Leiter des Zentrums für Kunst und Medientechnologie Karlsruhe) formulierte diese Erkenntnis bereits 2007, hob die Bedeutung des «User Generated Content» hervor und hinterfragte mit Blick auf Museen, ob diese weiterhin am Prinzip «Arche Noah» festhalten könnten und «sagen, wir haben die Definitionsmacht, wir können sagen und beurteilen, was Kunst ist, wir wählen aus, was Kunst ist, und Sie, die Betrachter/Besucher, dürfen ins Museum kommen und vor den von uns ausgewählten Kunstwerken auf die Knie fallen.» (ebd., 26)

Der Begriff des «User Generated Content» steht in engem Zusammenhang mit den Veränderungen der Netzkultur durch des Web 2.0 und die damit verbundene Entwicklung des Nutzers vom reinen Konsumenten zum Prosumenten, der durch vielfältige und leicht zu bedienende Tools selbst in die Lage versetzt wird, mediale Produktionen und damit auch Kunst hervorzubringen. Mit diesen Entwicklungen geht auch eine Veränderung im Bereich des Konsums einher (vor allem von Musik und Video). Die JIM-Studie 2018 belegt, dass mittlerweile 77\% aller Familien in Deutschland Videostreaming-Dienste wie Netflix oder Amazon Prime nutzen (vgl. mpfs 2018, 
7). Dies bringt auch Veränderungen im Konsumverhalten und der Konsumerwartung mit sich und zwar weg von der Linearität, der vorgegebenen Struktur des klassischen Fernsehens und Radios und hin zu einer selbstbestimmten On-Demand Kultur, welche individuelle Konsumentscheidungen zulässt. Für das Museum sah Weibel bereits 2007 die Gefahr, dass «[w]enn wir [...] weiter so verfahren wie ein Fernsehsender [...] und der Betrachter nicht die Möglichkeit hat, selbst ein Programm zusammenzustellen, dann wird das Museum obsolet.» (Weibel 2007, 26)

Vor diesem Hintergrund ist der Mehrwert einer Zusammenarbeit von Medienpädagogik und Kultureller Bildung von grösster Bedeutung. Gerade bezogen auf das Verständnis des Prozesses der Digitalisierung und den Umgang mit den dadurch entstehenden Potenzialen und Gefahren gibt es erhebliche Schnittmengen. Diese Schnittmengen zwischen Medienpädagogik und Kultureller Bildung werden auch in bildungspolitischen Forderungen und Empfehlungen aus beiden Richtungen deutlich (z.B. im Hinblick auf den Stellenwert aktiv-produktiver Lehr/Lern Szenarien sowie der Forderung im Rahmen des digitalen Wandels über mehr als nur technische Ausstattung zu sprechen). ${ }^{1}$

Mit einer Denkschrift zum Schwerpunktthema Kulturelle Bildung und Digitalisierung zeigt der Rat für Kulturelle Bildung, «welche Potenziale Kulturelle Bildung bereithält, um die Digitalisierung in der Schule zu gestalten.» (Rat für Kulturelle Bildung 2019b, 1). Es wird hervorgehoben, dass

«[d]er Umgang mit den Künsten [...] grundlegende Erfahrungen [ermöglicht] - wie die Schulung der Wahrnehmung, den Prozess des Auswählens oder den Umgang mit Vieldeutigkeit - die im Kontext der Digitalisierung immer wichtiger werden. Bei kreativen Kulturangeboten mit Musik, Tanz oder Video entwickeln Kinder und Jugendliche auch inhaltliches Interesse am Thema Digitalisierung.

1 Um diese Schnittmengen genauer zu betrachten, können zum Beispiel das Positionspapier «Technikausstattung alleine reicht nicht» der Initiative «Keine Bildung ohne Medien!»(https://www.keine-bildung-ohne-medien.de/technikausstattung-alleine-reicht-nicht/) und der Kurztext zur Denkschrift «Alles immer smart» vom «Rat für Kulturelle Bildung» (https://www.rat-kulturellebildung.de/fileadmin/user_upload/Kurztext_Denkschrift.pdf) gelesen werden. 
Wollen sie beispielsweise selbst im Internet produktiv sein, oder später in kreativen, kommunikativen oder den MINT-Bereichen arbeiten - auch hier geht es um neue Ästhetiken, Kulturen und Lebensentwürfe - brauchen Jugendliche Erfahrungen und Kenntnisse kultureller und ästhetischer Inszenierungs- und Gestaltungsprozesse. Diese werden mit Angeboten Kultureller Bildung traditionell erschlossen.» (ebd., l)

Diese Ausführungen sind anschlussfähig an die zum Beispiel von Niesyto skizzierten Potenziale einer handlungsorientierten Medienbildung, welche «das kreative Potenzial von Medien für Bildungs- und Lernprozesse freisetzen und Reflexionsprozesse im Umgang mit digitalen $\mathrm{Me}$ dien initiieren können» (Niesyto in Autenrieth et al. 2018, 152). Handlungsorientierung, das Aufgreifen lebens- und medienweltlicher Erfahrungen von Schülerinnen und Schülern sowie die Förderung aktiv-produktiver und kritisch-reflexiver Nutzung digitaler Medien sind wichtige Ziele für die Vermittlungsformate des Projekts RD. Unterstrichen werden die Projektziele auch durch die neusten Empfehlungen des Rats für Kulturelle Bildung, die das hohe Aktivierungspotenzial der audiovisuellen Medien aufgreifen. Sie regen an,

«die eigene Produktion Jugendlicher mit audiovisuellen Medien, [...] zu eigener künstlerischer Aktivität und Rezeption durch diese Medien sowie zur kritischen Reflexion der mit ihnen verbundenen Qualitäten und Realisierungsformen [zu fördern].» (Rat für Kulturelle Bildung 2019a, 8).

Unter dem Primat des Pädagogischen geht es bei der Ausgestaltung der ausserschulischen Erfahrungen im Projekt RD auch darum, die vielfältigen Potenziale des Digitalen zu nutzen und sie den beteiligten Schulen aufzuzeigen. Eingeordnet in das SAMR Modell nach Puentedura (siehe Abb. 1) will RD Szenarien entwickeln, die transformativen Charakter haben, in die Kategorie der «Redefinition» fallen und ohne Nutzung digitaler Medien gar nicht denkbar wären. 


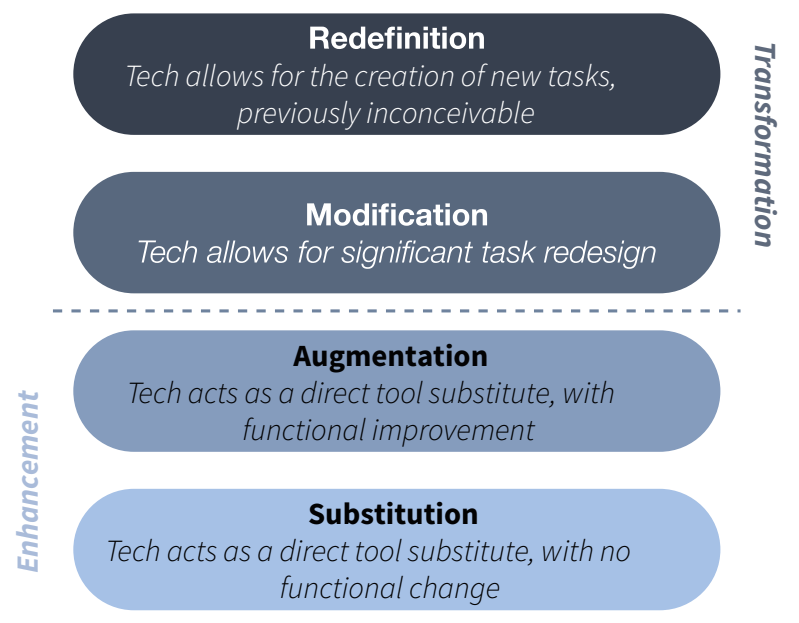

Abb. 1.: SAMR Modell nach Puentedura (vgl. www.hippasus.com/rrpweblog/).

Wie dringlich das Aufzeigen dieser qualitativ höherwertigen Standards ist, die unter Einsatz digitaler Medien erreicht werden können, verdeutlicht Thomas Knaus. Er hospitierte im Unterricht an fast 200 Schulen. Dabei stellte er fest, dass die Nutzung digitaler Medien im Regelunterricht selten aktiv-konstruierend, sondern meist passiv-rezeptiv erfolgt: zum Präsentieren, zur Recherche im Web oder zum Lesen von PDFs (vgl. Knaus 2018, 14). Dass diese Art von Tätigkeiten im Hinblick auf eine umfassende Medienkompetenzbildung unzureichend ist, zeigt die ICILS 2018 (International Computer and Information Literacy Study). Ähnlich wie schon 2013 belegt sie, dass 33,2\% der deutschen Achtklässlerinnen und Achtklässler (ein unverändert hoher Prozentsatz) nur rudimentäre, vorwiegend rezeptive Fähigkeiten und sehr einfache Anwendungskompetenzen vorweisen können und nur über basale Wissensbestände und Fertigkeiten hinsichtlich der Identifikation von Informationen und der Bearbeitung von Dokumenten verfügen (vgl. Eickelmann und Labusch 2019, 8). Das heisst, dass einem Drittel der Jugendlichen in Deutschland ein selbstbestimmter und partizipativer Umgang mit den Möglichkeiten der Entwicklungen der Digitalisierung grösstenteils verwehrt bleiben. Beachtet man nun den weitgehenden Konsens, dass Medienkompetenz zu den zentralen Schlüsselkompetenzen des 21. Jahrhunderts gehört (vgl. Eickelmann et al. 2014, 4) muss ein Projekt wie RD auch das Problem dieser digitalen Spaltung berücksichtigen. 
Verständig et al. betrachteten dieses Problem 2016 als «weitgehend unstrittig», dass die Nutzung der Potenziale und Möglichkeiten des Internets «ungleich verteilt sind und sich gerade auch sozial-strukturelle Ungleichheiten im Internet reproduzieren» (Verständig et al. 2016, 51). Auch ICILS 2018 belegt solche herkunftsbedingten Disparitäten (vgl. Eickelmann und Labusch 2019, 7). Um eine möglichst grosse Breitenwirksamkeit zu entfalten und zu einer chancengerechten Medienbildung für alle Schülerinnen und Schüler beizutragen liegt daher ein weiterer Schwerpunkt von RD in einem Beitrag zur Förderung der medienpädagogischen Kompetenz angehender Lehrkräfte wobei auch hier die besonderen Chancen eines Einbezug ausserschulischer Möglichkeits- und Erfahrungsräume und deren Erschliessung durch digitale Medien eine Schlüsselposition einnehmen. Der Bedarf dafür ergibt sich unter anderem auch aus einer Absolventenbefragung an den fünf Pädagogischen Hochschulen in Baden-Württemberg aus dem Jahr 2018. Diese zeigt, dass 72\% der Absolventinnen und Absolventen das Ausmass der Förderung von EDV-Kenntnissen und Medienkompetenz im Rahmen des Studiums mit «eher zu wenig» oder «zu wenig» bewerten (vgl. Statistisches Landesamt Baden-Württemberg 2019, 47).

Die bisherigen Ausgangsüberlegungen zum Projekt machen deutlich, dass die Zusammenarbeit von Schule (Schülerinnen und Schüler, Lehrkräfte, Schulleitungen), Hochschule (Studierende und Dozierende) und ausserschulischen Bildungseinrichtungen wie Museen (Leitung und pädagogisches Personal) zur Entstehung erheblicher Mehrwerte für alle Beteiligten beitragen kann und dies mit einem hohen Partizipationsgrad dieser einhergeht.

\section{2 «Mit» statt «Für»: Das Projekt RD als partizipatives Projekt kultureller Teilhabe}

Von Beginn des Projekts RD an sind die Zielgruppen, die Schülerinnen und Schüler, die Lehramtsstudierenden und Lehrkräfte unmittelbar am Projekt beteiligt, und zwar auf Augenhöhe. Doch was bedeutet das im Einzelnen? Welche Rolle etwa nehmen die Schülerinnen und Schüler ein? Sind sie lediglich Konsumenten eines von Fachleuten konzipierten Bildungsangebots, das sie aufnehmen - und im besten Fall - verinnerlichen? Oder haben 
sie wirklich Teil an der Entwicklung und Ausgestaltung des Projekts, sind aktiv-produktiv einbezogen und damit lernbereit, wissensdurstig und neugierig (vgl. Hüther 2013, 25)? Die amerikanische Autorin Nina Simon unterscheidet in ihrer Publikation «The Participatory Museum» fünf Stadien der «Begegnung» von Museumsbesucherinnen und -besuchern und Museum (vgl. Simon 2010, 1 ff.). Die Partizipationstreppe (vgl. Abbildung 2) beginnt beim rein konsumierenden Museumsaufenthalt eines Einzelbesuchers oder einer Einzelbesucherin. Von Stufe zu Stufe nimmt der Grad der Involviertheit der Besucherin oder des Besuchers mit den Museumsinhalten zu, indem sich unter verschiedenen Aufgabenstellungen zu den Ausstellungsstücken und Themen im Museum verhalten werden kann. Man eignet sich die über die Exponate transportierten Themen produktiv an, kreiert im Idealfall eine eigene Ausstellung und begibt sich dabei in einem zunehmend intensiven Austausch mit anderen Museumsbesucherinnen und -besuchern, oder Museumsmitarbeiterinnen und -mitarbeitern, die Ideen und Ansichten teilen oder auch nicht, so dass ein veritabler Diskurs entsteht. Mit diesen Stadien einher geht eine Bewegung vom Ich zum Wir, aus der Einzelperspektive hin zu einer Multiperspektivität, vom Individuum zum Miteinander. Im fünften und höchsten Stadium ist das Museum ein Ort, an dem das Publikum weitaus mehr verhandelt als nur die vorgegeben Museumsinhalte. Im Idealfall entwickeln die Besucherinnen und Besucher das Museum weiter, aktiv und kreativ. Legt man diese Massstäbe und Skalierung an das Projekt RD an, erreicht das Modellprojekt den höchsten Grad von Partizipation und damit der als Projektziel beschriebenen kulturellen Teilhabe einer jüngeren Generation durch: die integrativen, kooperativen und künstlerisch-produktiven Entwicklungs- und Aneignungsprozesse. Für Pforzheim und möglicherweise weit darüber hinaus könnte Reuchlin digital als Museumsprojekt mit hohem partizipativem Wirkungsgrad und Transferpotenzial zum Vorbild werden. 


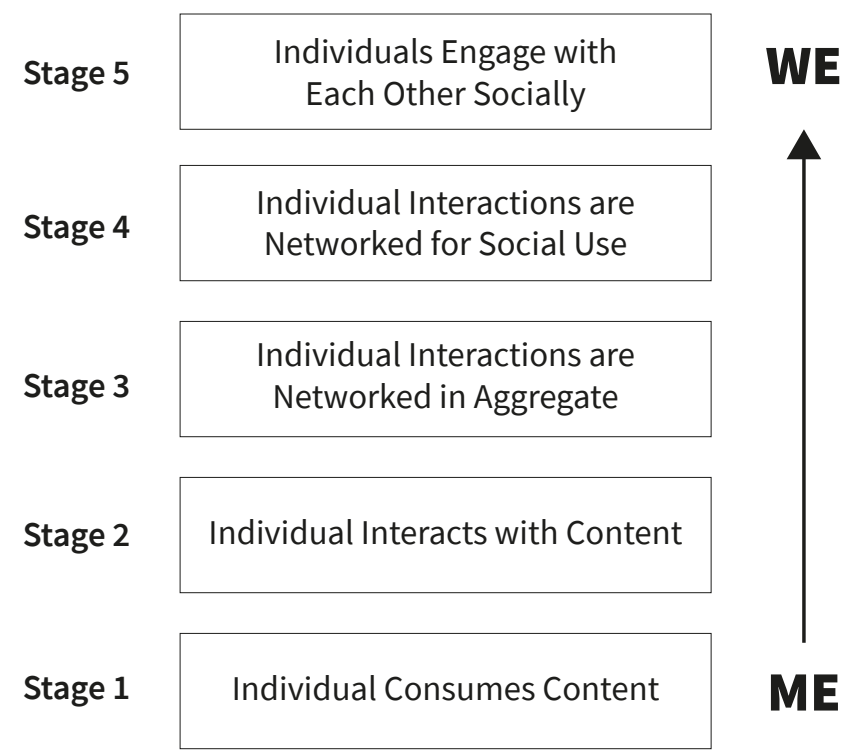

Abb. 2.: Die fünf Stufen sozialer Partizipation (Simon 2010).

\subsection{Auf dem Weg zu kultureller Teilhabe: Kulturelle Bildung im Verständnis des Kulturamts Pforzheim}

Das MJR ist eines von sieben städtischen Museen, an denen das Kulturamt Pforzheim Angebote Kultureller Bildung etabliert hat. Die Kulturelle Bildung ist seit 2013 ein zentrales Handlungsfeld kommunaler Kulturarbeit in Pforzheim. Sie richtet sich vor allem an Kitas und Schulen, um möglichst vielen Kindern der Stadt Teilhabe an Kultur und Bildung zu ermöglichen. Denn Kulturelle Bildung ist ein Menschenrecht aller Kinder und Jugendlichen, wie es in der allgemeinen Erklärung der Menschenrechte und in der Kinderrechtskonvention der Vereinten Nationen sowie im Grundgesetz und in den Länderverfassungen verbrieft ist. (vgl. BKJ 2015, 4)

«Kunst, Kultur, Spiel und Medien sind spezifische Ausdrucksformen des Menschen. Mit ihrer Hilfe erschließen sich Kinder und Jugendliche die Welt. Sie lernen nicht nur, die Welt zu verstehen, sondern auch zu reflektieren und zu gestalten. Sie entwickeln ihre Identität und finden ihre Position. ... Kulturelle Bildungsangebote geben den Bedürfnissen von Kindern und Jugendlichen nach Selbstentfaltung, 
Mitgestaltung und Gemeinschaft Raum. Zugleich unterstützen sie jungen Menschen dabei, Kompetenzen wie Kreativität, Kritikfähigkeit, Selbstbewusstsein, Toleranz und Verantwortungsbereitschaft zu entwickeln.» (BKJ 2015, 5)

Kulturelle Bildung trägt somit massgeblich zur Entwicklung jener 21st century skills (creativity, cooperation, communication, critical reflection) bei, die als massgeblich für die Bewältigung anstehender Zukunftsaufgaben gelten (vgl. Applied Educational Systems (AES) o. J.). Und «Kulturelle Bildung ermöglicht [...] gesellschaftliche, politische und kulturelle Beteiligung.» (BKJ 2015, 5)

Bildung mit Lebensweltbezug und Alltagslernen wird in den aktuellen Bildungsplänen von Kita und Schule durchaus thematisiert. Gerade für Kinder und Jugendliche aus zugewanderten Familien, denen das Traditionswissen zum neuen Wohnort fehlt, spielt sie eine wichtige Rolle. Deshalb bildet die Orientierung in Raum und Zeit einen zentralen Fokus der Kulturellen Bildung in Pforzheim. Orientierung schafft Sicherheit und ist eine Grundvoraussetzung für kulturelle Teilhabe und Identifikation. Viele Kinder und Jugendliche aus Pforzheim stammen aus bildungsbenachteiligten Familien. Sie verfügen nicht über Wissen um das eigene Umfeld und haben kaum oder keine Teilhabe am kulturellen und gesellschaftlichen Leben. Das ist keineswegs ausschliesslich dem hohen Migrationsanteil in der Pforzheimer Stadtgesellschaft geschuldet, sondern auch einer problematischen Sozialstruktur, deren Ursachen mannigfaltig sind. Orientierung erfahren die Kinder und Jugendlichen in den Angeboten Kultureller Bildung nicht nur in Raum und Zeit, sondern auch im Hinblick auf das gesellschaftliche Miteinander, auf die Werte und Normen, denen unsere freiheitlichdemokratischen Grundordnung verpflichtet ist. Auch sie sind Thema der Auseinandersetzung, gerade und explizit im MJR.

Das Kulturamt vertritt in seinen Angeboten eine ausdrückliche Bedarfs- und Stärkenorientierung. Jedes Kind, jede/r Jugendliche wird dort abgeholt wo es/er/sie steht und wird an seinen/ihren Stärken gemessen (vgl. Hüther 2013, 110). Der Umgang erfolgt auf Augenhöhe. Auftrag der Kulturellen Bildung in den Museen Pforzheims ist in erster Linie Kompetenzförderung und nicht Wissensvermittlung. Stichwort: Empowerment, 
um zu einen selbstbestimmten, selbstgestalteten, glücklichen Leben zu gelangen. Dabei liegt ein weites Bildungsverständnis zugrunde, das emotional-affektive, kognitiv-intellektuelle, körperlich-sinnliche und sozialkulturelle Prozesse umfasst (vgl. BKJ 2015, 5).

In Veranstaltungen der Kulturellen Bildung in Pforzheim steht vernetztes und selbst motiviertes Lernen im Fokus, ebenso Selbstwirksamkeitserfahrungen, Selbstvergewisserung und Persönlichkeitsentwicklung. Eine wichtige Rolle spielt dabei die Förderung von Schlüsselkompetenzen (vgl. Wenzlik 2013). Sie werden unterschieden nach Selbstkompetenzen (z.B. Belastbarkeit, Eigeninitiative, Flexibilität), Sozialkompetenzen (z.B. Einfühlungsvermögen, Teamfähigkeit, Kritikfähigkeit) und Methodenkompetenzen (z.B. Problemlösefähigkeit, Reflexionsfähigkeit). (vgl. ebd.)

In der Vermittlung dieser Kompetenzen und in der Begleitung der damit verbundenen Prozesse verstehen sich die Mitarbeiterinnen und Mitarbeiter aus dem Team Kulturelle Bildung des Kulturamts Pforzheim im Sinne Gerald Hüthers als Potentialentfaltungcoachs (vgl. Hüther 2013, 30,109). Ihr Ziel ist es, den Kindern und Jugendlichen neue Weltzugänge an besonderen, authentischen Orten, unter Einsatz der ästhetischen und kreativen Potentiale der Künste, zu eröffnen. Allerdings haben die Medien und insbesondere digitale Formate mit Ausnahme der Fotografie bisher keine Berücksichtigung im Programmangebot gefunden.

\subsection{Das MJR als ausserschulischer Lernort}

Seit der Eröffnung des Museums im Jahr 2008 hat das Kulturamt interessierten Schulklassen klassische Führungen durch das Museum angeboten. 2013 wurden Vermittlungsformate im Sinne der teilhabeorientierten Kulturellen Bildung erweitert. Seitdem rücken Lebensweltbezug der Schülerinnen und Schüler und Handlungsorientierung in den Mittelpunkt. In Kleingruppen erkundeten die Schülerinnen und Schüler mit Arbeitsblättern das Museum. Im abschliessenden Plenum wurden die Ergebnisse gesammelt und gesichert. Allerdings war früh zu konstatieren, dass diese sehr sprach- und textzentrierte Herangehensweise für die hohe Zahl von Schülerinnen und Schülern mit nicht vorhandenen oder begrenzten 
Sprachkenntnissen in Deutsch nicht geeignet war. Auch für diese Schülerinnen und Schüler sollten langfristig andere Zugänge zum MJR geebnet werden.

Trotz zahlreicher Werbemassnahmen und direkter Ansprachen persönlich bekannter Schulleitungen und Lehrkräfte war die Resonanz von Schulklassen auf das Bildungsangebot im Museum unbefriedigend. Im Durchschnitt besuchten zehn Schulklassen pro Jahr das MJR, mehrheitlich aus Gymnasien. Auf Rückfrage bei verschiedenen Schularten und Lehrkräfte wurden unterschiedliche Gründe genannt. Eine Auswahl sei hier angeführt: der «schwierige Stoff», den das Museum vermittle und der sich nicht für alle Schularten eigne; der Zeitdruck, den Bildungsplan durchzuarbeiten, der keinen Raum für ausserschulische Vertiefung oder Ergänzung zulasse; die fragliche Relevanz der Themen im MJR, die für den Horizont der Schülerinnen und Schüler zu weit entfernt wären; mangelnder Lebensweltbezug.

Mit dieser Ausgangssituation sah sich das Kulturamt konfrontiert, als es 2018 den Entschluss fasste, sich neuen Vermittlungszugängen zuzuwenden. Der Innovationsfonds des Landes Baden-Württemberg hatte eine neue Förderlinie für innovative digitale Vermittlungsprojekte aufgelegt, auf die sich das Kulturamt als Träger des MJR mit dem Projektkonzept «Reuchlin digital. Eine digitale Plattform am Lernort Museums Johannes Reuchlin» beworben hat. Die Bewerbung war erfolgreich, das Projekt wurde bewilligt und startete offiziell im Oktober 2018. Zeitgleich sicherte die Arbeitsstelle für literarische Museen, Archive und Gedenkstätten in Baden-Württemberg (ALIM) einen Förderbeitrag zu. Bereits im Vorfeld galt es, zahlreiche Weichen zu stellen, Projekt- und Kooperationspartner auszuwählen, ein interdisziplinäres Projektteam und einen Lenkungskreis zusammenzustellen, in dem sich die unterschiedlichen Kompetenzbereiche, die in der Projektenwicklung notwendig waren und sind, widerspiegeln: Kulturelle Bildung mit Museumspädagogik, Medienpädagogik, Medienwissenschaft, Softwareentwicklung, Literaturwissenschaft, Kulturwissenschaft, Gaming, Pädagogik, Fachdidaktik in Geschichte, Gemeinschaftskunde und Ethik. 


\subsection{In Schleifen zum Ziel: Design Thinking als Projektansatz}

Bereits bei der ersten Zusammenkunft entschied sich der Lenkungskreis gegen die noch im Antragskonzept verfochtene lineare Projektentwicklungsstruktur. Stattdessen plädierte er auf Empfehlung mehrerer Expertinnen und Experten in der Runde für einen Paradigmenwechsel und für ein agiles, iteratives Projektverfahren, das Elemente aus dem Design Thinking (vgl. Lewrick u. a. 2018, 10 ff.) aufnimmt (vgl. Abb. 3).

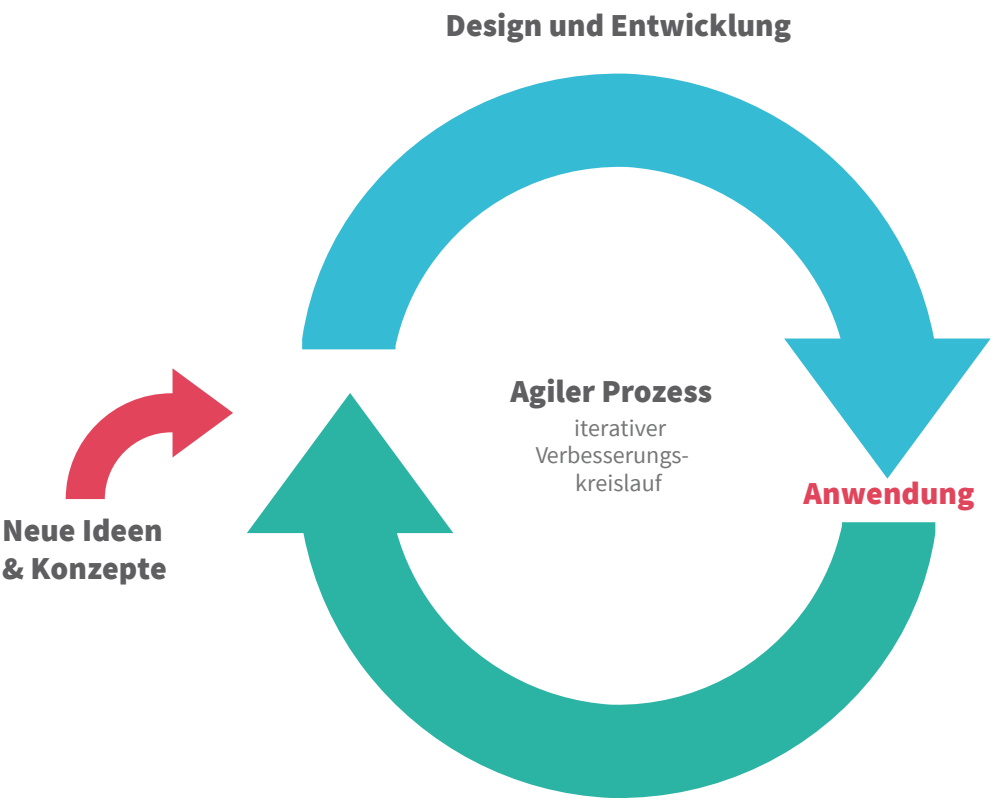

Erprobung \& Feedback

Abb. 3.: Projektprozess basierend auf agilen und Design Thinking Strukturen.

Verkürzt dargestellt ist Design Thinking ein strukturierter Problemlösungsansatz der darauf abzielt, mit einem Team von Menschen aus unterschiedlichen Disziplinen - in einem kreativen Umfeld - nutzerfreundliche Ideen und Innovationen zu gestalten und umzusetzen. Das können materielle Produkte sein oder, wie in unserem Fall, eine digitale Vermittlungsplattform für das MJR. Design Thinking basiert auf Kreativmethoden des Industrial Design. Dieses Verfahren wurde Anfang der goer Jahre in den USA in modifizierter Form auf die Software-Entwicklung übertragen. 
Inzwischen hat der Ansatz, der darauf gründet, nicht ausschliesslich nur das grosse Ganze (den Makroprozess) mit dem Endziel im Blick zu haben, sondern in kleineren Entwicklungszyklen (sogenannten Mikroprozessen) Ideen möglichst frühzeitig in Form von Prototypen «greifbar» werden zu lassen, weltweite Verbreitung gefunden. Ausgangsvoraussetzung ist eine genaue Analyse der Nutzerinnen und Nutzer, in die sich die Projektpartner idealerweise «einfühlen», um deren Bedürfnisse und Motivationen zu Verstehen. In einem weiteren Schritt des Beobachtens werden die Nutzerinnen und Nutzer (je nach Umfeld auch Kundinnen und Kunden) näher betrachtet, wobei zumeist qualitative Erhebungsmethoden eingesetzt werden. Beim Standpunkt definieren beleuchtet die Gruppe die gesammelten Ergebnisse im intensiven Erfahrungsaustausch, mit dem Ziel, die im Fokus stehenden Nutzerinnen und Nutzer noch detaillierter zu erfassen. Im nächsten Schritt wird im Brainstorming das Ideen Finden angeregt mit möglichst vielen Konzepten und Visualisierungen. Dieses Stadium ist Voraussetzung für das Prototypen entwickeln und das damit verbundene Testen durch die Nutzerinnen und Nutzer, an dessen Schlusspunkt meist ein qualitatives Feedback steht. Für jede Phase gibt es drei Handlungsoptionen: Love it, change it, or leave it. Votieren die Nutzerinnen und Nutzer für die zweite Option, gibt es eine erneute, ggf. leicht verkürzte Schleife, in der nachjustiert wird, während die nächste Idee parallel dazu von Grund auf neu entwickelt wird, sodass die einzelnen Zyklen zeitgleich nebeneinander her laufen können: Dabei ist ein zentraler Erfolgsfaktor zu wissen, wo man im Prozess steht. Weitere Erfolgsfaktoren sind neben einem interdisziplinären Team der nötige Spielraum für Experimente, Kreativität und Mut, Bestehendes zu hinterfragen.

\subsection{Ziele des Projekts Reuchlin digital: Eine Annäherung in Schleifen}

Mit dem für viele Projektbeteiligte neuen Projektmanagment-Tool des Design Thinking ist das Projekt Reuchlin digital sehr schnell in ein produktives und konstruktives Fahrwasser gelangt. Es wurde zum Teil kontrovers diskutiert, mit welcher Absicht und Motivation Schülerinnen und Schüler in das Museum kommen (Verstehen). In der ersten Projektphase Anfang 
Dezember 2018 wurden daher fünf «Forschergruppen» (51 Schülerinnen und Schüler im Alter zwischen 13 und 21 Jahren) - die ausdrücklich ohne Vorkenntnisse sein sollten - zur Erkundung ins MJR eingeladen und nach ihren Eindrücken und Wahrnehmungen befragt. Je zwei Schülerinnen und Schüler wurden von einer museumspädagogischen Fachkraft begleitet (Betrachten). Beim Besuch des Museums ging es nicht um Wissensvermittlung, sondern um selbstgesteuerte Raumerfahrung und daraus abgeleitete Lernmotivation. Ein Fragebogen erfasste zudem die ersten Eindrücke, Lieblingsplätze und Lieblingsobjekte sowie die Wünsche bezüglich weiterer Museumsbesuche. Zentrale Erkenntnisse waren unter anderem das starke inhaltliche Interesse der Schülerinnen und Schüler, konkrete Hinweise auf gewünschte Vermittlungsformen (zum Beispiel «Erklärvideos, wie auf YouTube»), der Wunsch nach mehr interaktiven Angeboten sowie das Bedürfnis nach Führung (nicht zwangsläufig im traditionellen Sinn durch Museumsführerinnen oder Museumsführer). Die Auswertung der Fragebögen und die Diskussion der Ergebnisse setzte neue Schwerpunkte, auch was die inhalts- und prozessbezogenen Kompetenzen betrifft, die im MJR gefördert werden sollen (Standpunkt definieren). Danach herrschte unter den Projektbeteiligten Einigkeit, dass RD in erster Linie darauf ausgerichtet ist, den Ort des MJR in die mentalen Landkarten (vgl. u.a. Downs und Stea 1982) der Schülerinnen und Schüler einzuschreiben und mit dem Museum einen Lernraum für eigenständige und handlungsorientierte Wissensaneignung zu schaffen. Eine weitere zentrale Komponente der Auseinandersetzung der Schülerinnen und Schüler mit dem MJR ist das Thema Medienbildung und die Aneignung von Medienkompetenz in Zeiten digitaler Transformationsprozesse. Denn das Lebenswerk Reuchlins eignet sich als thematischer Anker, um den Metaprozess der Digitalisierung beziehungsweise Mediatisierung besser zu verstehen und gleichzeitig Werte- und Kompetenzdiskussionen zu Themen wie Freiheit, Würde, Mitmenschlichkeit usw. anzustossen. Ziel ist es daher auch, analoge Faceto-Face Vermittlung mit der kreativen Gestaltung interaktiver Aufgaben zu verknüpfen, um neue, ganzheitliche und lebensweltbezogene Lernprozesse mit hoher Eigenmotivation und Selbststeuerung in Gang zu setzen. Erste Prototypen für neue Vermittlungsformate (Prototypen entwickeln) entstanden aus der Kooperation mit der Pädagogischen Hochschule Ludwigsburg. Diese werden im Folgenden genauer betrachtet. 


\subsection{Projektevaluation}

$\mathrm{RD}$ ist angelegt als praxisorientiertes Entwicklungsprojekt. Der Projektfokus liegt daher auf der Konzeption und Erprobung neuer handlungsorientierter Vermittlungsformate für das MJR. Um Gelingensbedingungen, Mehrwerte, Lernpotenziale aber auch Stolpersteine herauszuarbeiten, wird projektintern eine formative Evaluation durchgeführt. Das Projektteam einigte sich dafür auf folgende Leitfragen ${ }^{2}$ :

- Wie werden museale und unterrichtsbezogene sowie lebens- und medienweltliche Themen in die aktiv-produktive Gestaltung mit digitalen Medien am ausserschulischen Lernort MJR einbezogen?

- Inwieweit werden altersangemessene, kritisch-reflexive Aspekte in die aktiv-produktive Gestaltung mit digitalen Medien integriert und von den Schülerinnen und Schüler aufgenommen?

- Welche Erfahrungen machen Museumsmitarbeiterinnen und -mitarbeiter, Schulleitungen, Jugendliche, Lehrkräfte, Studierende und Dozierende im Projektverlauf?

- Inwieweit gelingt die Umsetzung der geplanten Vermittlungsformate?

Folgende Methoden werden bei der Evaluation eingesetzt:

- Einzel- und Gruppeninterviews mit Projektpartnern (Schülerinnen und Schüler, Studierende, Lehrkräfte, Schulleitungen) zu projektbezogenen Erfahrungswerten und durch das Projekt entstandenen Mehrwerten

- Fragebögen für Schülerinnen und Schüler zur Ermittlung soziodemografischer und medienbezogener Informationen

- phänomenologisch orientierte Vignettenforschung (vgl. Mayer-Drawe 2010, 6) sowie strukturierte Auswertungen medialer Artefakte.

2 Die Leitfragen und Methoden orientieren sich am Evaluationskonzept des Entwicklungsprojekts dileg-SL (Digitales Lernen Grundschule - Stuttgart/ Ludwigsburg) welches von 2016 bis 2019 an der Pädagogischen Hochschule Ludwigsburg und der Rosensteinschule in Stuttgart durchgeführt wurde (vgl. Niesyto 2019a, 32). Der Fokus des Projekts dileg-SL lag zum einen auf der Aneignung von Kompetenzen für den pädagogisch-didaktisch sinnvollen Einsatz digitaler Medien durch Lehramtsstudierende sowie in der Entwicklung konkreter handlungsorientierter Unterrichtseinheiten unter Verwendung verschiedener medialer Aneignungs- und Darstellungsformen (vgl. Niesyto 2019b, 208). 


\section{Neue Vermittlungsformate im Museum}
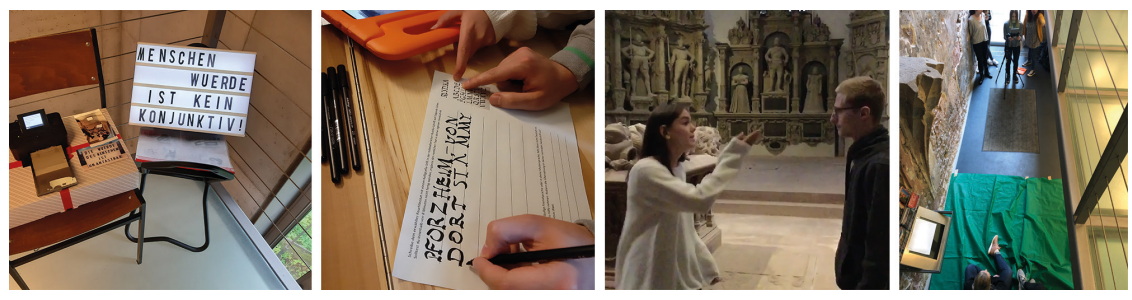

Abb. 4.: Schülerinnen und Schüler bei der Medienproduktion im Museum Johannes Reuchlin, gemeinsam mit Studierenden und Dozierenden der $\mathrm{PH}$ Ludwigsburg und Mitarbeiterinnen des Kulturamts Pforzheim.

Erste konkrete und an einem umfassenden Medienbegriff orientierte Vermittlungsformate (für Schülerinnen und Schüler ab Klassenstufe 7/8) für RD entstanden aus der Kooperation mit der PH Ludwigsburg. In einem praxisorientierten Blockseminar hatten die Lehramtsstudierenden die Aufgabe, handlungsorientierte und selbstgesteuerte Lehr-Lern-Settings zu konzipieren, dabei museumsbezogene Inhalte aufzugreifen und so zur Medienkompetenzbildung von Schülerinnen und Schüler im Rahmen der Erprobung dieser Konzepte im Museum beizutragen. Nach der Erarbeitung medienpädagogischer Grundlagen, vor allem im Hinblick auf Prinzipien aktiver Medienarbeit (vgl. u.a. Demmler und Rösch 2012) und der Erkundung des MJR vor Ort hatten die Studierenden selbst die Möglichkeit, sich in einer Seminarsitzung mit Workshopcharakter mit verschiedenen Apps, Online Tools und Geräten vertraut zu machen und den Umgang damit einzuüben. Aus den Erfahrungen des Entwicklungsprojekts dilegSL (Digitales Lernen Grundschule - Stuttgart/Ludwigsburg), an dem die Dozierenden des hier beschriebenen Seminars beteiligt waren, ergab sich die Notwendigkeit der Vermittlung von Basiskompetenzen im produktivgestalterischen Umgang mit digitalen Medien sowie auch technisch-gestalterischer und mediendidaktischer Kompetenzen(vgl. Junge et al. 2019, 320). Mit diesem theoretischen und praktischen Hintergrundwissen gestalteten die Studierenden, unter Einbezug der Ergebnisse aus der Schülerinnen-und-Schüler-Befragung, verschiedene Prototypen, welche mit Hilfe eines Tools zur Gestaltung interaktiver und multimedialer Touren (Actionbound) zur Verfügung gestellt wurden (vgl. Abbildung 5). Auf diese 
Weise konnten die Schülerinnen und Schüler als Zielgruppe der Vermittlungsformate gruppenweise selbst entscheiden, welche Themen sie interessieren und welche Aufgaben sie bearbeiten möchten. Einige Aufgaben sowie Medienproduktionen der Schülerinnen und Schüler sollen hier exemplarisch vorgestellt werden. Aufgabenintentionen und aus den Erprobungen resultierende Ergebnisse werden anschliessend erläutert.

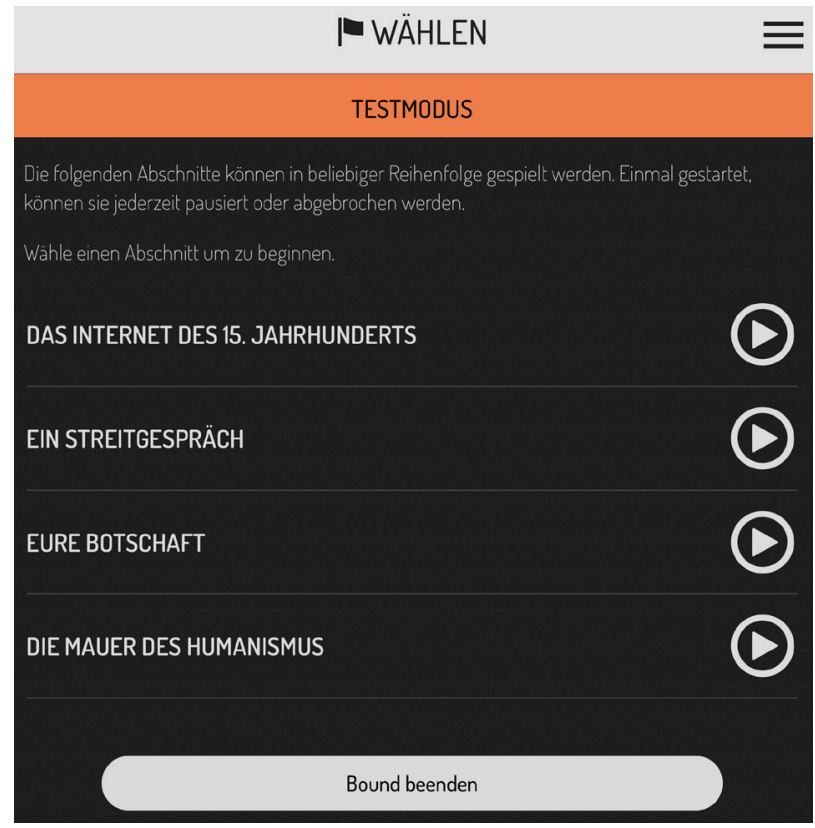

Abb. 5.: Aufgabenübersicht. 


\subsection{Das Internet des 15. Jahrhunderts}

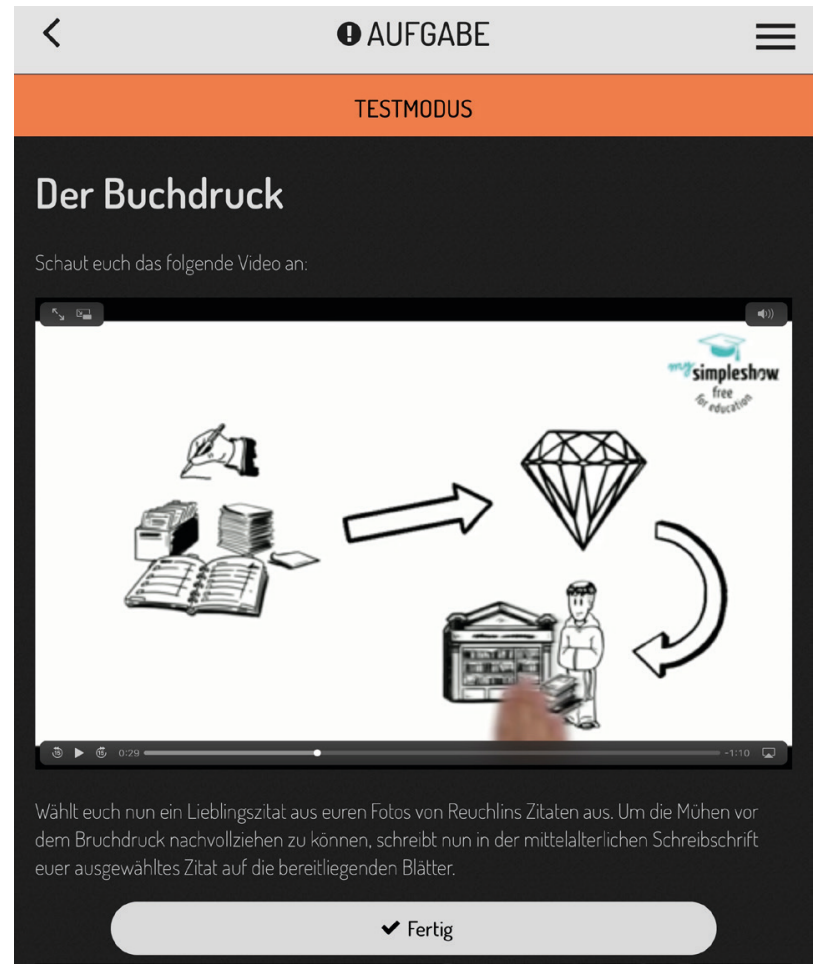

Abb. 6.: Von Studierenden entwickeltes Erklärvideo zum Thema Buchdruck.

Um die Medienrevolution des Buchdrucks für Schülerinnen und Schüler zugänglich zu machen, erhielten die Schülerinnen und Schüler über Anweisungen in Actionbound zunächst die Aufgabe, das MJR nach Zitaten Reuchlins zu durchsuchen und diese in geeigneter Form (zum Beispiel als Foto) festzuhalten. Anschliessend begaben sie sich auf die Suche nach einem im Umfeld eines Druckerpresse-Modells platzierten QR Code. Dieser führte die Schülerinnen und Schüler zu einem von den Studierenden produzierten Erklärvideo. Das etwa zweiminütige Video vermittelte den Schülerinnen und Schüler einen prägnanten Überblick zur Bedeutung des Buchdrucks und vollzog durch einen Vergleich mit der Bedeutung des Internets den Brückenschlag zum Titel der Aufgabe («Das Internet des 15. Jahrhunderts»). Um den revolutionären Charakter des Buchdrucks 
erfahrbar zu machen, wurde die Aufgabe dadurch abgeschlossen, dass die Schülerinnen und Schüler eines der fotografierten Zitate von Johannes Reuchlin in einer mittelalterlichen Schreibschrift mit einem Kalligrafiestift auf Papier notierten. Als Zusatzaufgabe sollte dieses Zitat anschliessend in eine der in der Gruppe vertretenen Muttersprachen übersetzt werden. Falls in einer Gruppe ausschliesslich Deutsch als Muttersprache vertreten sein sollte, wurde die Option zur Übersetzung auf Englisch angeboten. Zur Unterstützung, unabhängig von der gewählten Sprache, wurde auf ein Übersetzungstool hingewiesen.

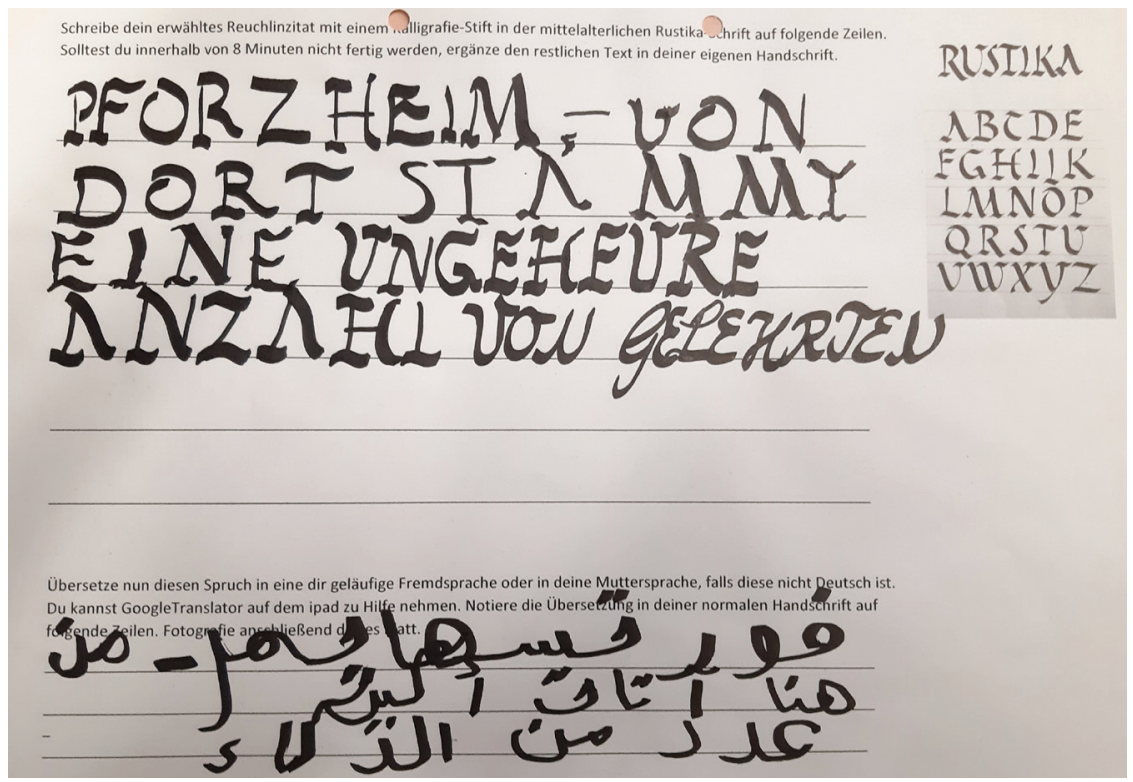

Abb. 7.: Beispiel Handschrift und Übersetzung.

\subsection{Die Mauer des Humanismus}

Die heute vom Lauf der Geschichte erheblich gezeichnete südliche Aussenmauer des Schlosskirchenchores, an die im 15. Jahrhundert die Sakristei mit Reuchlins Bibliothek und über 500 Jahre später, 2008, das MJR angebaut wurde, ist das einzige bauliche Originalfragment aus Reuchlins Zeit innerhalb des eigentlichen Museums. Das Projektteam hat diese authentische, heute inwändige Mauer als «Mauer des Humanismus» umfirmiert. 
Die Bedeutung der Mauer im Kontext Reuchlins bleibt den meisten Schülerinnen und Schüler zunächst verschlossen. Über Aufgaben in der Actionbound App werden sie aufgefordert, im Museum nach Informationen zu suchen, was das MJR an dieser Stelle zu einem authentischen Ort macht. Die Informationen werden zusammengetragen und in einem kurzen Video aufgezeichnet. Dabei editieren sich die Schülerinnen und Schüler mithilfe einer Videoaufzeichnung vor einem Greenscreen in die Mauer des Humanismus. Sie interagieren und werden - zumindest virtuell - ein Teil von ihr (siehe Abbildung 8).
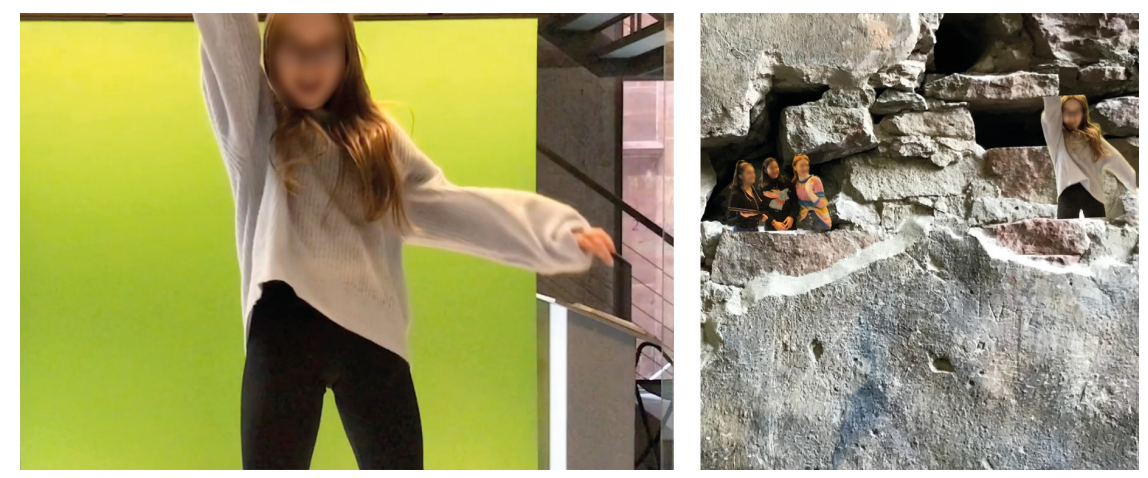

Abb. 8.: Neue Zugangsform zur Mauer des Humanismus mittels Greenscreen Technik.

\subsection{Aufgabenintentionen und erste Erfahrungswerte}

Die beiden oben skizzierten Aufgaben geben einen Einblick in die Art der Vermittlungsformate, die von den am Projekt beteiligten Studierenden erarbeitet und mit Schülerinnen und Schüler erprobt wurden. Diese Formate wurden und werden im Projektverlauf auf der Basis der laufenden formativen Evaluation weiterentwickelt und durch weitere Vermittlungsformate ergänzt. Zum jetzigen Zeitpunkt kann allerdings schon festgehalten werden, dass die bisherigen Formate die Schülerinnen und Schüler auf vielfältige Art und Weise berührt haben. So eröffnet beispielsweise die Aufgabe «Die Mauer des Humanismus» einen ganz neuen Zugang zum eher intellektuell geprägten Begriff des authentischen Orts. Durch die Arbeit mit digitalen Medien entsteht zudem eine andere Art der Orts- und Raumwahrnehmung. Vielfältige Reflexionsanlässe zur Entwicklung der 
Medienkritikfähigkeit (vgl. Niesyto 2018, 59) der Schülerinnen und Schüler bot insbesondere die Arbeit mit Greenscreenaufnahmen. Diese Arten der digitalen Gestaltungstechniken schaffen mit ihrer Eigendynamik einen neuen Umgang mit dem Realen, Sinnlich-Körperlichen, UnmittelbarAuthentischen. So zeigt die Mauer unter anderem auch Zerstörungs- und Verfallsspuren. Bereits die Aufnahme eines kleinen Ausschnitts und dessen Isolierung in einem Foto ist eine Form der Wahrnehmung als ungewöhnliche Begegnung. Die Erstellung von Bewegungsaufnahmen und deren Implementierung in einen neuen Kontext ermöglichen diskursive Auseinandersetzungen. Spannungsfelder in Bezug auf den historischen Kontext eröffnen sich. Das Unplanbare liegt im kreativen Zusammenspiel von unterschiedlichen Personen und der Auseinandersetzung mit der historischen Mauer, dem sichtbaren Ergebnis und damit verbundenen Tätigkeiten, Experimenten, einprägsam-erinnernden ästhetischen Erfahrungen, sowie Irritationen und Provokationen.

Auf der Basis von Schülerinnen-und-Schüler-Befragungen im direkten Anschluss an die Erprobung konnte unter anderem festgestellt werden, dass durch die Aufgabe «Das Internet des 15. Jahrhunderts» trotz der teilweise bereits bekannten Fakten zum Buchdruck erst jetzt das Ausmass dieser kulturverändernden Technik erkannt und ein neuer Bezug zum Medium Schrift und Buch hergestellt werden konnte. Als überaus motivationssteigernd erwies sich ein Teilaspekt der Aufgabe, nämlich die Transkription des Zitats von Johannes Reuchlin in die Muttersprache, wie das Beispiel eines arabischstämmigen Schülers erkennen liess. Anders als im Regelunterricht, beteiligte er sich auffällig stark auch an den nachfolgenden produktiven Aufgaben. Hier zeigt sich die Bedeutung und nachhaltige Wirkung von Stärkenorientierung als Grundprinzip Kultureller Bildung (vgl. Stute und Wibbing 2014).

\section{Zwischenbilanz}

Zum jetzigen Zeitpunkt (November 2019) waren 30 Studierende der Pädagogischen Hochschule Ludwigsburg aus den Studiengängen für das Lehramt, und den Bildungswissenschaften aktiv an der Entwicklung von RD beteiligt. Es konnten 70 Schülerinnen und Schüler von vier Schulen (zwei 
neunte Klassen des Reuchlin Gymnasiums, eine gymnasiale Kursstufe 1 der Carlo Schmid Schule und eine achte Klasse der Karl Friedrich Werkrealschule) sowie vier Lehrkräfte der beteiligten Schulen in die Entwicklung und Erprobungen der neuen digitalen Vermittlungsformate einbezogen werden. Mit einer der neunten Klassen des Reuchlin Gymnasiums wurden zudem weitere Erklärvideos (zu verschiedenen Themen wie Humanismus ${ }^{3}$ oder Pforzheim im Mittelalter) produziert. Diese finden in unterschiedlichen Kontexten aber vor allem im Rahmen selbstgesteuerter Lernerfahrungen im MJR Verwendung. Die Nachhaltigkeit des Projekts basiert damit primär auf dem Erfolgsfaktor Kooperation und der damit erreichten interdisziplinären Vernetzung einerseits, andererseits auf der Einbeziehung der Zielgruppen in die Projektentwicklung. Diese Herangehensweise ermöglicht es langfristig, das Projekt ohne kostenintensive Leistungen kommerzieller Agenturen zu realisieren. Die Fördermittel fliessen aktuell mehrheitlich in «Produkte» und Anwendungen, an denen die Kooperationspartner je nach Aufgabe in unterschiedlichen Konstellationen mitwirken. So wird mittel- und langfristig ein weit gespanntes und belastbares Netzwerk etabliert, das auch nach Beendigung des Förderzeitraums im September 2020 weiterbestehen und garantieren wird, dass Reuchlin digital mit moderaten Budgets weiterentwickelt werden kann. Die digitale Transformation der Vermittlungsarbeit im MJR ist damit kein einmaliges Unterfangen mit einem feststehenden Endergebnis, sondern ein Prozess, der im Sinne von Nachhaltigkeit, Partizipation und agilem Projektmanagement andauert und weitergeführt werden wird.

Für die am Projekt beteiligten Schülerinnen und Schüler, Studierende und Lehrkräfte konnten durch die partizipativ und auf eine Zusammenarbeit auf Augenhöhe angelegte Projektstruktur bereits jetzt zahlreiche Mehrwerte geschaffen werden. So konnten unter anderem die Studierenden durch die Auseinandersetzung mit dem Lernort MJR kontextuale medienpädagogische Gestaltungen erproben und Methoden der Kulturellen Bildung kennenlernen. Ausgehend vom authentischen und inspirativen Museumsort haben sie vielfältige Lehr-Lern-Szenarien unter Einbezug digitaler Medien entwickelt und ortsspezifisch ausgestaltet (siehe oben

3 Einen exemplarischen Einblick bietet ein Video zum Thema Humanismus welches hier abgerufen werden kann: https://diopdalwg490s.cloudfront.net/ fileadmin/user_upload/kultur/reuchlin_digital/IMG_0087.mp4. 
skizzierte Aufgaben). Gleichzeitig haben sie die Bedeutung ausserschulischer Lernorte unter pädagogisch-didaktischen Gesichtspunkten kennengelernt. Die Studierenden erlebten unmittelbare Rückmeldungen von ihrer Zielgruppe. Hier fand eine direkte Auseinandersetzung mit den entwickelten Ideen und des Feedbacks der Jugendlichen statt. Von den Schülerinnen und Schülern wurden insbesondere die aktiv-produktiven Möglichkeiten in der Auseinandersetzung mit visuellen, auditiven und audiovisuellen Ausdrucksformen hervorgehoben. Exemplarisch dafür ist folgendes Zitat aus der Befragung von Schulklassen im Anschluss an eine Erprobungsphase: «Sonst wird einem nur was erzählt. So konnte man was Kreatives selber machen und es sich besser merken.» (Schülerin einer zehnten Klasse)

Die Schülerinnen und Schüler erlebten nicht nur vielfältige Facetten der Medienkompetenzentwicklung (Mediengestaltung, Medienkritik, selbstbestimmter Umgang, aktive Kommunikation, etc.). Die aktive Einbindung in das Projekt ermöglichte ihnen neue Selbstwirksamkeitserfahrungen, vor allem im Wissen darüber, dass ihre Rückmeldungen die Projektentwicklung entscheidend beeinflussen und ihre Produktionen (zum Beispiel Erklärvideos) im Projekt aktive Verwendung finden. Die bei den Befragungen der Schülerinnen und Schüler festgehaltene Aussage, «Super, dass wir unsere Meinung sagen durften!», dokumentiert die Bedeutung dieser Form von Kooperation und Partizipation, die echte kulturelle Teilhabe ermöglicht. Die bisherigen Erfahrungen deuten darauf hin, dass museale und unterrichtsbezogene sowie lebens- und medienweltliche Themen sowie die aktiv-produktive Gestaltung mit digitalen Medien am ausserschulischen Lernort MJR einbezogen werden können und entsprechende Mehrwerte sichtbar werden. Die folgende Aussage aus einem Gespräch mit einer beteiligten Schülerin zeigt stellvertretend dafür, dass sowohl kreativ-gestalterische Aspekte als auch Wahlfreiheit bei der Auswahl von Themen und Aufgaben von den Schülerinnen und Schülern positive Zuschreibungen erfahren: «Sonst wird einem im Museum nur was erzählt. So konnte man selber etwas machen und es sich viel besser merken.» Die Evaluationsfrage, inwieweit kritisch-reflexive Aspekte in der Nutzung und Gestaltung mit digitalen Medien von den Schülerinnen und Schülern aufgenommen werden, kann in der aktuellen Projektphase noch nicht abschliessend beantwortet werden. 
Aus den Interviews mit aussenstehenden Lehrkräften, die nicht am Projekt beteiligt waren, wurde jedoch deutlich, dass an den meisten Schulen mehrheitlich eine grosse Unsicherheit im Umgang mit digitalen Medien besteht und weder Ausstattung noch ausreichend pädagogischdidaktisches Wissen und Erfahrungen zum Einsatz dieser Medien vorhanden ist. Dennoch wurde die Konrektorin einer Werkrealschule durch die Beobachtung ihrer Schulklasse im aktiv-produktiven Umgang mit Tablets (im Rahmen von RD Vermittlungsformaten) dazu ermutigt, an der Schule vorhandene, bislang nicht genutzte Tablets im Unterricht zu RD einzusetzen. Zwei Lehrpersonen eines Gymnasiums erklärten sich bereit, Ihre Klassen als Testklassen das MJR mit entsprechenden Feedback-Aufgaben besuchen zu lassen. Damit sind inzwischen von fünf befragten Lehrkräften drei neue Lehrkräfte (6o Prozent) in das Projekt involviert, nachdem sie sich in ausführlichen und zeitaufwändigen Gesprächen über das Projekt und seine Potenziale haben informieren und «anstecken» lassen.

Als gravierender Stolperstein für das Projekt ergab sich der enge Zeitrahmen, den die meisten Lehrkräfte anmahnen: Lediglich 90-120 Minuten sind für den Museumsbesuch akzeptabel. Anfängliche Vorstellungen, einen Schulvormittag lang mit den Schülerinnen und Schülern im Museum arbeiten zu können, erwiesen sich als Utopie, auch vor dem Hintergrund, dass es in Pforzheim kaum Ganztagesschulen gibt, deren Curricula für solche Angebote aufgeschlossener sind. Diese Rahmenbedingung wurden in den Vermittlungsangeboten berücksichtigt. Aktuell werden auf den Museumsbesuch vorbereitende und nachbereitende Angebote (Unterrichtsmaterialien zur thematischen Vorbereitung und vor allem zur (Medienpädagogischen)Reflexion des Besuchs) konzipiert, sodass die aktive $\mathrm{Me}$ dienarbeit einen Schwerpunkt des Museumsbesuchs einnehmen kann und die Schülerinnen und Schüler während des Museumsbesuchs genügend Zeit für das Produzieren und Experimentieren haben. Es bleibt zu hoffen, dass der Regelbetrieb von RD derart nachhaltige qualitative Ergebnisse zeigt, dass ein Umdenken seitens der Lehrerschaft erfolgt und RD mit seinem Mehrwert wahrgenommen und auf adäquate Weise mit den lokalen Schulcurricula verknüpft wird. 
Die bislang im Projekt erworbene Expertise des Projektteams wird bereits von der Medien- und Filmgesellschaft Baden-Württemberg (im Projekt «Museen im Wandel») und von der Arbeitsstelle für literarische Museen, Archive und Gedenkstätten in Baden-Württemberg im Rahmen von Workshops, Tagungen und Webinaren angefragt. Beide Einrichtungen attestieren RD den Status als innovatives Modellprojekt über die Stadtgrenzen Pforzheims hinaus, mit einem hohen Potenzial an Transferwissen, das sich auf andere, mittlere und kleinere Museen mit überschaubaren Budgets übertragen lässt.

\section{Ausblick}

In der nächsten Projektphase werden weitere Erprobungen durchgeführt auf deren Basis weitere Anpassungen/Verbesserungen an den Vermittlungsformaten vorgenommen werden. Als weitere Zugangsform zum Museum wird das Thema Gamification in den Blick genommen. Eine Anregung, die ebenfalls von den Schülerinnen und Schülern der Forscherklassen eingebracht wurde. Im Wintersemester 2019/2020 werden Studierende der PH Ludwigsburg Konzepte für die Entwicklung eines Escape Rooms in den Räumlichkeiten des MJR entwickeln und diese mit Schulklassen erproben. Dabei gilt es zu untersuchen, ob die Systematik und Methodik des Escape Rooms auf den musealen Raum übertragen werden kann, welche Rolle digitale Medien dabei spielen können und wie die Verbindung von Historie, Pädagogik und Game gelingend umsetzbar wird. Erste konzeptionelle Bemühungen im Rahmen des Hochschulseminars sowie Expertengespräche zwischen Studierenden, Lehrenden und Game Designern deuten auf innovative Potenziale für das museale Erleben aber auch im Hinblick auf persönlichkeitsbezogene und soziale Kompetenzen hin.

\section{Literatur}

Applied Educational Systems (AES). o. J. «What Are 21st Century Skills?». https:// wWw.aeseducation.com/career-readiness/what-are-21st-century-skills.

Assmann, Aleida. 2017. Menschenrechte und Menschenpflichten: auf der Suche nach einem neuen Gesellschaftsvertrag: Vortrag im RadioKulturhaus Wien am 22. Juni 2016. Wiener Vorlesungen, Band 188. Wien: Picus Verlag. 
Autenrieth, Daniel, Horst Niesyto, Katrin Schlör, und Anja Marquardt. 2018. «Digitales Lernen in der Grunschule - ein Werkstattbericht mit Praxisbeispielen aus dem Projekt dileg-SL». In Spannungen und Potentiale: Digitaler Wandel in Bildungseinrichtungen, von Thomas Knaus, herausgegeben von Olga Engel. München: Kopaed.

Bundesvereinigung Kulturelle Kinder- und Jugendbildung (BKJ). 2015. «Kulturelle Bildung ist Koproduktion. Außerschulische und schulische Kulturelle Bildung wirksam entfalten». KULTURELLE BILDUNG ONLINE. https://www. kubi-online.de/artikel/kulturelle-bildung-koproduktion-ausserschulischeschulische-kulturelle-bildung-kinder.

Demmler, Kathrin, und Eike Rösch. 2012. «Aktive Medienarbeit in Zeiten der Digitalisierung». In Medienpädagogik Praxis Handbuch, von Elisabeth JäckleinKreis, herausgegeben von Tobias Albers-Heinemann. München: Kopaed.

Downs, Roger M., und David Stea. 1982. Kognitive Karten: Die Welt in unseren Köpfen. Herausgegeben von Robert Geipel. New York: UTB.

Eickelmann, Birgit, Stefan Aufenanger, und Bardo Herzig. 2014. «Medienbildung entlang der Bildungskette. Ein Rahmenkonzept für eine subjektorientierte Förderung von Medienkompetenz im Bildungsverlauf von Kindern und Jugendlichen». Deutsche Telekom Stiftung. https://www.telekom-stiftung.de/ sites/default/files/files/media/publications/buch_medienbildung.bildungskette_end.pdf.

Eickelmann, Birgit, und Amelie Labusch. 2019. ICILS 2018 \#Deutschland auf einen Blick. Münster: Waxmann. https://kw.uni-paderborn.de/fileadmin/fakultaet/ Institute/erziehungswissenschaft/Schulpaedagogik/ICILS_2018__Deutschland_Presseinformation.pdf.

Gesellschaft für Medienpädagogik und Kommunikationskultur (GMK). 2018. «Medienbildung in der Schule ist mehr als digitale Bildung - Medienpädagogik positioniert sich zur Strategie der Kultusministerkonferenz „Bildung in der digitalen Welt"». https://www.gmk-net.de/wp-content/uploads/2018/og/ gmk-positionspapier_kmk-strategie_27092018.pdf.

Hüther, Gerald. 2013. Kommunale Intelligenz: Potenzialentfaltung in Städten und Gemeinden. Hamburg: Ed. Körber-Stiftung.

Jörissen. 2016. «Hegemoniale Ästhetiken und ästhetische Gegenstrategien in der postdigitalen Kultur». https://joerissen.name/wp-content/uploads/2017/02/ Jörissen-Benjamin-2016-preprint.-Hegemoniale-Ästhetiken-und-ästhetische-Gegenstrategien-in-der-postdigitalen-Kultur.pdf.

Jörissen, Bejamin. 2013. «Medienbildung〉 in 5 Sätzen». Education \& Culture. 2013. https://joerissen.name/medienbildung/medienbildung-in-5-satzen/.

Knaus, Thomas. 2018. «[Me]nsch - Werkzeug - [I]nteraktion. Theoretisch-konzeptionelle Analysen zur 〈Digitalen Bildung〉 und zur Bedeutung der Medienpädagogik in der nächsten Gesellschaft». MedienPädagogik: Zeitschrift für Theorie Und Praxis Der Medienbildung 31 (〈Digitale Bildung〉), 1-35. https://doi. org/10.21240/mpaed/31/2018.03.26.X. 
Krotz, Friedrich. 2007. Mediatisierung: Fallstudien zum Wandel von Kommunikation. 1. Aufl. Medien, Kultur, Kommunikation. Wiesbaden: VS, Verlag für Sozialwissenschaften.

Kultusministerkonferenz. 2012. «Medienbildung in der Schule». https://www.

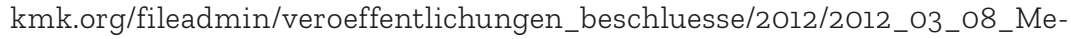
dienbildung.pdf.

Lewrick, Michael, Patrick Link, Larry Leifer, und Nadia Langensand, Hrsg. 2018. Das Design Thinking Playbook: mit traditionellen, aktuellen und zukünftigen Erfolgsfaktoren. 2., überarbeitete Auflage. München: Verlag Franz Vahlen GmbH.

Mayer-Drawe, Käte. 2010. «Zur Erfahrung des Lernens. Eine phänomenologische Skizze». Santalka 18(3),.

Medienpädagogischer Forschungsverbund Südwest (mpfs). 2018. «JIM-Studie 2018. Jugend, Information, Medien». https://www.mpfs.de/fileadmin/files/ Studien/JIM/2018/Studie/JIM2018_Gesamt.pdf.

Niesyto, Horst. 2018. "Medienkritik - Entwicklungslinien und aktuelle Herausforderungen». In Medienkritik im digitalen Zeitalter, herausgegeben von Heinz Moser und Horst Niesyto. Medienpädagogik interdisziplinär 1l. München: Kopaed.

Niesyto, Horst. 2019a. «Digitales Lernen Grundschule - Ausgangsüberlegungen, Ziele und Strukturen des Entwicklungsprojekts dileg-SL». In Digitale Medien in der Grundschullehrerbildung Erfahrungen aus dem Projekt dileg-SL, von Thorsten Junge, herausgegeben von Horst Niesyto, Kopaed. München.

Niesyto, Horst. 2019b. «Ergebnisse des Entwicklungsprojekts dileg-SL. Kernpunkte in teilprojektübergreifender Perspektive». In Digitale Medien in der Grundschullehrerbildung Erfahrungen aus dem Projekt dileg-SL, von Thorsten Junge, herausgegeben von Horst Niesyto, Kopaed. München.

Niesyto, Horst, Thorsten Junge, und Robert Rymes. 2019. «Überlegungen zur Nachheiltigkeit des Entwicklungsprojekts dileg-SL». In Digitale Medien in der Grundschullehrerbildung Erfahrungen aus dem Projekt dileg-SL, von Thorsten Junge, herausgegeben von Horst Niesyto, Kopaed. München.

Puentedura, Ruben R. o. J. «SAMR: A Brief Introduction». Zugegriffen 2. Dezember 2019. http://hippasus.com/rrpweblog/archives/2015/10/SAMR_ABriefIntro. pdf.

Rat für Kulturelle Bildung. 2019a. «Jugend / Youtube / Kulturelle Bildung. Horizonte 2019». https://www.rat-kulturelle-bildung.de/fileadmin/user_upload/ pdf/Studie_YouTube_Webversion_final.pdf.

Rat für Kulturelle Bildung. 2019b. «Kurztext zur Denkschrift zum Schwerpunktthema "Kulturelle Bildung und Digitalisierung". Alles immer smart Kulturelle Bildung, Digitalisierung, Schule». https://www.rat-kulturelle-bildung.de/ fileadmin/user_upload/Kurztext_Denkschrift.pdf.

Schwab, Hans-Rüdiger. 1998. Johannes Reuchlin: Deutschlands erster Humanist: ein biographisches Lesebuch. Orig.-Ausg. dtv 12609. München: Dt. TaschenbuchVerl.

Simon, Nina. 2010. The Participatory Museum. Santa Cruz, California: Museum 2.0. 
Stadt Pforzheim. 2019. «Pforzheimer Bevölkerung 2018. Teil 1: Bevölkerungsentwicklung und Struktur». https://diopdalwg49os.cloudfront.net/fileadmin/ user_upload/statistik/2019/Bevölkerungsheft_2018_-Teill.pdf.

Statistisches Landesamt Baden-Württemberg. 2019. «Ergebnisse der Absolventenbefragung 2018 an den Pädagogischen Hochschulen in Baden-Württemberg». https://www.ph-ludwigsburg.de/fileadmin/subsites/gi-verw-t-ol/user_ files/Startseite/2019/2018_PH_Absolventenbefragung_Gesamtbericht.pdf.

Sturm, Patrick. 2018. «Johannes Reuchlin - Werk, Bedeutung, Rezeption. Neue Ausstellung im Lesesaal des Stadtarchivs». Herausgegeben von Stadtarchiv Pforzheim. Archivmagazin 2018/3.

Stute, Dirk, und Gisela Wibbing. 2014. "Kulturelle Bildung als Baustein der Unterrichtsentwicklung». KULTURELLE BILDUNG ONLINE. https://www.kubionline.de/artikel/kulturelle-bildung-baustein-unterrichtsentwicklung.

Timm, Christoph. 2018. «Zehn Jahre Museum Johannes Reuchlin». Herausgegeben von Freunde der Schlosskirche. Blickpunkt. Schlosskirche Blickpunkt. Schlosskirche 2018/2019 (28).

Verständig, Dan, Alexandra Klein, und Stefan Iske. 2016. «Zero-Level Digital Divide: Neues Netz und neue Ungleichheiten». Si:So. Analysen - Berichte - Kontroversen, 50-55.

Weibel, Peter. 2007. «Web 2.0 und das Museum». In Vom Betrachter zum Gestalter: neue Medien in Museen-Strategien, Beispiele und Perspektiven für die Bildung, herausgegeben von Michael Mangold, Peter Weibel, und Julie Woletz, 1. Aufl. Baden-Baden: Nomos.

Wenzlik, Alexander. 2013. «Schlüsselkompetenzen in der Kulturellen Bildung». KULTURELLE BILDUNG ONLINE. https://www.kubi-online.de/artikel/schluesselkompetenzen-kulturellen-bildung. 